\title{
The Metal/Electroactive Polymer Interface Studied by Surface Resistance
}

\author{
Ricardo Tucceri \\ Instituto de Investigaciones Fisicoquímicas Teóricas y Aplicadas (INIFTA), Consejo Nacional de Investigaciones Científicas y \\ Técnológicas (CONICET), Facultad de Ciencias Exactas, Universidad Nacional de La Plata, La Plata, Argentina. \\ Email: rtucce@gmail.com \\ Received May $6^{\text {th }}, 2013$; revised June $7^{\text {th }}, 2013$; accepted June $27^{\text {th }}, 2013$ \\ Copyright (C) 2013 Ricardo Tucceri. This is an open access article distributed under the Creative Commons Attribution License, \\ which permits unrestricted use, distribution, and reproduction in any medium, provided the original work is properly cited.
}

\begin{abstract}
The experimental arrangement in this investigation was one in which a poly $(o$-aminophenol) (POAP) film was supported on a thin gold film electrode whose thickness is of the order of the mean free path of conduction electrons of gold. This arrangement allows one to apply the surface resistance technique to study the electrochemical processes occurring at the metal film surface coated with the polymer film. The dependence of the resistance change of the thin gold film electrode on the external electrolyte composition for polymer thickness lower than $0.25 \mathrm{mC} \cdot \mathrm{cm}^{-2}$, was attributed to a competition, at the gold film surface, between the redox process of the polymer and adsorption of different ion species contained in the electrolyte. This competition reflects a discontinuous character of polymer thickness lower than 0.25 $\mathrm{mC} \cdot \mathrm{cm}^{-2}$ at the metal polymer interface. The resistance response of the metal film becomes independent of both the external electrolyte composition and polymer thickness for polymer thickness higher than $0.8 \mathrm{mC} \cdot \mathrm{cm}^{-2}$. Then, POAP thicknesses higher than $0.8 \mathrm{mC} \cdot \mathrm{cm}^{-2}$ seem to be compact enough at the metal polymer interface to prevent the interaction of the species contained in the supporting electrolyte with the gold film surface. The increase of the gold film resistance in going from the reduced to the oxidized state for POAP thicknesses higher than $0.8 \mathrm{mC} \cdot \mathrm{cm}^{-2}$ was attributed to the redox conversion of poly (o-aminophenol) from amine to imine groups. This resistance increase was explained as a transition from specular to diffuse scattering of conduction electrons of gold at the gold poly (o-aminophenol) interface due to a less compact distribution of oxidised sites of POAP as compared with that of the reduced ones. An attenuation of the resistance response of the gold film was observed when the POAP films were deactivated either by contact with a ferric cation solution or prolonged potential cycling. The deactivation of the polymer film was attributed to the creation of inactive gaps within the redox sites configuration of POAP. The surface resistance technique allows one to detect different redox sites configurations of POAP on the gold film, according to the method used to deactivate the polymer films. In this work, it is demonstrated that the surface resistance technique can be employed to study not only the ability of a POAP film to inhibit the interaction of different species in solution with a metal surface but also the deactivation of the polymer film.
\end{abstract}

Keywords: Surface Resistance; Thin Gold Film Electrode; Poly(o-Aminophenol) Films; Poly(o-Aminophenol) Gold Interface; Poly(o-Aminophenol) Deactivation

\section{Introduction}

The surface resistance (SR) technique is very sensitive to the surface scattering of the conduction electrons caused by species present on a metal film surface $[1,2]$. The technique was used to study different electrode processes [3-7]. In this work, we study the surface resistance and voltammetric responses of a gold film electrode coated with POAP in the presence of different ion species in solution. Two external variables, the electrolyte composition and the polymer thickness, were changed. The main objectives of this work are, firstly, to study how the interaction of ion species contained in the electrolyte solution with the gold surface is affected by the gradual coverage of the metal surface with the polymer film; secondly, if the own redox process of the POAP film affects the surface resistance of the metal film; and finally, if the deactivation of the POAP film deposited on the gold film can be monitored by surface resistance. Adsorption of different ion species on naked metal film surfaces has been extensively studied by SR $[3,4]$. With regard to the 
redox process of POAP films synthesized in acid medium, it was studied employing different electrochemical techniques [8-17] and was attributed to the conversion from amine to imine groups [12]. While voltammetry allows one to characterise quantitatively the redox species transformation of the polymer, surface resistance allows one to enquire about changes in the electronic properties at the gold film polymer film interface during the redox conversion of the polymer. POAP films were also deactivated by employing two different methods; the first one by soaking the POAP films in a ferric cation solution [18] and the second one, by prolonging potential cycling in the supporting electrolyte solution. As the surface resistance technique may be considered as a non-traditional approach in the electrochemistry of polymers, interesting additional information about the redox conversion of POAP at the metal polymer interface and the polymer deactivation is obtained in this work.

\section{Experimental}

\subsection{The Gold Film Electrodes}

Gold thin film electrodes of constant thickness $\phi_{\mathrm{m}} \sim 30$ $\mathrm{nm}$ were prepared by vacuum evaporation as it was described previously [4]. These gold films are polycrystalline with crystallite sizes between 0.01 and $0.1 \mu \mathrm{m}$ [5]. The samples could be inserted into an electrochemical cell similar to that showed in a previous paper [6] and employed as working electrodes. An electrode area of 1 $\mathrm{cm}^{2}$ was exposed to the solution. These electrodes exhibit initial resistance values $R \sim 20 \mathrm{ohms}$. The relationship between the length $l$ and the width $w$ of these gold film electrodes was $G=l / w=2$. A gold grid of large area was used as counter electrode. All the potentials reported in this work are referred to the SCE.

The gold film conductivity was measured by circulating a $d c$ current $(1 \mathrm{~mA})$ between the extreme contacts while polarising the electrode ensemble through the central contact [7]. The resistance change obtained in this way was recorded as a function of the potential $(\Delta R / R-E)$ together with the voltammetric response $(j-E)$. The experimental set-up for simultaneous voltammetric and surface conductivity measurements on thin film electrodes has been described in detail previously $[3,4,6]$. The effect of different anions on the resistometric response of gold was studied from $0.4 \mathrm{M} \mathrm{NaClO}_{4}+0.1 \mathrm{M} \mathrm{HClO}_{4}, 0.4 \mathrm{M}$ $\mathrm{Na}_{2} \mathrm{SO}_{4}+0.1 \mathrm{M} \mathrm{H}_{2} \mathrm{SO}_{4}$ and $0.4 \mathrm{M}$ sodium benzenesulphonate $+0.1 \mathrm{M}$ benzenesulphonic acid solutions, respectively. The effect of a cation, such as $\mathrm{Cu}(\mathrm{II})$, able to be deposited on gold under upd conditions [19], was studied from a $0.1 \mathrm{M} \mathrm{HClO}_{4}+10^{-4} \mathrm{M} \mathrm{Cu}\left(\mathrm{ClO}_{4}\right)_{2}$ solution.

\subsection{The POAP Coated Gold Film Electrodes}

POAP coated gold film electrodes were obtained by immersing the gold films described in the previous section in a $10^{-3} \mathrm{M}$ orthoaminophenol $+0.4 \mathrm{M} \mathrm{NaClO}_{4}+0.1 \mathrm{M}$ $\mathrm{HClO}_{4}$ solution and cycling the potential between -0.25 $\mathrm{V}$ and $0.8 \mathrm{~V}$ at a scan rate $v=0.05 \mathrm{~V} \cdot \mathrm{s}^{-1}$. These experimental conditions lead to adherent POAP films on the gold film surface, mechanically stables and with a very reproducible electrochemical response [8-10]. The POAP thickness was increased by increasing the number of potential cycles in the last solution containing the monomer. In order to obtain a measure of the polymer thickness, these POAP coated gold film electrodes were then rinsed and transferred to the supporting electrolyte solution (0.4 $\left.\mathrm{M} \mathrm{NaClO}{ }_{4}+0.1 \mathrm{M} \mathrm{HClO}_{4}\right)$. Then, the voltammetric reduction charge $Q_{\text {Red }}$ in this last solution was determined by integrating the cathodic current of the voltammetric response between $-0.25 \mathrm{~V}$ and $0.5 \mathrm{~V}\left(v=0.05 \mathrm{~V} \cdot \mathrm{s}^{-1}\right)^{26}$. After this, $Q_{\text {Red }}$ was considered as repre- sentative of the polymer thickness. Five polymer thickness $Q_{\text {Red }}(\sim 0.12$, $0.22,0.42,0.9$ and $1.5 \mathrm{mC} \cdot \mathrm{cm}^{-2}$ ), respectively, were employed. Many workers have correlated for this type of polymers, the charge obtained from the integration of the voltammetric profile to the ellipsometric thickness [10, 15]. Although this procedure is reasonable within an order of magnitude, the ellipsometric thickness is usually obtained under the assumption of a homogeneous film that is, averaging the optical film properties across its thickness. As our resistance data evidence heterogeneity of thin POAP films $\left(Q_{\text {Red }}<0.25 \mathrm{mC} \cdot \mathrm{cm}^{-2}\right)$ in the presence of different electrolytes (see below), in this work the voltammetric charge is quoted, which should be related, although not directly, to the polymer film thickness. According to a previous work [10], the $Q_{\text {Red }}$ values above indicated would correspond to $\phi_{\mathrm{p}}$ values around $2,5,12$, 22 and $40 \mathrm{~nm}$, respectively. With these POAP coated gold film electrodes resistance responses were recorded (separately for each $\phi_{\mathrm{p}}$ value) within the potential range $-0.2 \mathrm{~V}$ and $0.5 \mathrm{~V}$ at a scan rate $v=0.01 \mathrm{~V} \cdot \mathrm{s}^{-1}$ in solutions containing $\mathrm{ClO}_{4}^{-}, \mathrm{SO}_{4}^{-2}$, and benzenesulphonate $\left(\mathrm{C}_{6} \mathrm{H}_{5} \mathrm{SO}_{3}^{-}=\mathrm{BS}\right)$ anions, respectively. Also, $\Delta R / R$ changes were obtained in the presence of a $\mathrm{Cu}$ (II) solution. Before record these responses, POAP coated gold electrodes were cycled in the different electrolytes for at least $1 \mathrm{~h}$.

Deactivation experiments were performed with two series of 8 electrodes each one. The first series of 8 films was deactivated by soaking them in a ferric cation solution and the second one was deactivated by prolonged potential cycling. With regard to the first series, each POAP film was successively employed in an individual experiment similar to that described in [16-18]. That is, each POAP film, after being equilibrated within the po- 
tential region $-0.2<E<0.5 \mathrm{~V}$ in the supporting electrolyte solution, was soaked in a $0.1 \mathrm{M} \mathrm{H}_{2} \mathrm{SO}_{4}+0.05 \mathrm{M}$ $\mathrm{Fe}_{2}\left(\mathrm{SO}_{4}\right)_{3}$ solution for different time periods (see second column in Table 1). Then, each one of these electrodes was extracted from the solution containing ferric ions and it was copiously washed with the supporting electrolyte solution, then the electrode was again transferred to the electrochemical cell containing the supporting electrolyte solution. The corresponding $j-E$ and $\Delta R / R$ responses were again recorded for each one of the 8 POAP films.

With regard to the second series of 8 films, each POAP film was subjected to a different number of potential cycles (higher than 500 cycles) within the potential region $-0.2<E<0.5 \mathrm{~V}$ in a deoxygenated supporting electrolyte solution (see first and second columns of Table 2). Then, the corresponding $j-E$ and $\Delta R / R$ responses were again recorded for each one of the 8 POAP films. In all cases, an attenuation of the voltammetric and surface resistance responses after deactivation of the films was observed. POAP films with $Q_{\text {Red }}$ values about of 2.8 $\mathrm{mC} \cdot \mathrm{cm}^{-2}$ were employed in deactivation experiments.

\subsection{Apparatus, Chemical and Solutions}

A PAR Model 173 potentiostat together with a PAR Model 175 function generator were used. The potential drop at the extremes of the film was measured with a Keithley Model 160 voltmeter during the resistometric measurements. A X-Y $Y_{1}-Y_{2}$ Hewelett Packard Model 7046 $\mathrm{B}$ plotter was used for simultaneous records of $\Delta R / R-E$ and $j-E$ responses.

Table 1. Voltammetric reduction charge $Q_{\text {Red,c }}$ and degree of deactivation, $\theta_{c}$, of different POAP films after contact with a ferric cation solution.

\begin{tabular}{cccc}
\hline POAP films $^{\mathrm{a}}$ & Soaking time $^{\mathrm{b}} / \mathrm{h}$ & $Q_{\text {Red,c }}{ }^{\mathrm{c}} / \mathrm{mC} \cdot \mathrm{cm}^{-2}$ & $\theta_{\mathrm{c}}^{\mathrm{d}}$ \\
\hline 1 & 2 & 2.44 & 0.13 \\
2 & 4 & 2.04 & 0.27 \\
3 & 5 & 1.87 & 0.33 \\
4 & 10 & 1.57 & 0.44 \\
5 & 16 & 1.32 & 0.53 \\
6 & 21 & 1.15 & 0.59 \\
7 & 24 & 1.12 & 0.60 \\
8 & 33 & 1.06 & 0.62 \\
\hline
\end{tabular}

${ }^{\mathbf{a}}$ Numbers 1 to 8 represent different deactivated POAP films. ${ }^{\mathbf{b}}$ Different soaking times in a $0.1 \mathrm{M} \mathrm{H}_{2} \mathrm{SO}_{4}+0.05 \mathrm{M} \mathrm{Fe}_{2}\left(\mathrm{SO}_{4}\right)_{3}$ solution. ${ }^{\mathrm{c}}$ Voltammetric reduction charge of the different deactivated POAP films after being subjected to the soaking process in the presence of the ferric cation solution. ${ }^{\mathbf{d}}$ Degree of deactivation of the different POAP films calculated from $\theta_{\mathrm{c}}=1-$ $\left(Q_{\text {Red,c }} / Q_{\text {Red,T }}\right) \cdot Q_{\text {Red,T }}\left(=2.8 \mathrm{mC} \cdot \mathrm{cm}^{-2}\right)$ is the voltammetric reduction charge of a non-deactivated film.
Table 2. Effect of the number of potential cycles on the surface resistance change of a POAP-coated gold film electrode.

\begin{tabular}{ccccc}
\hline${ }^{\mathrm{a}}$ Film & $\begin{array}{c}{ }^{\mathrm{b}} \text { Number of } \\
\text { potential cycles }\end{array}$ & ${ }^{\mathrm{c}} Q_{\text {Red, } \mathrm{c}} / \mathrm{mC} \cdot \mathrm{cm}^{-2}$ & ${ }^{\mathrm{d}} \theta_{\mathrm{c}}^{\mathrm{d}}$ & ${ }^{\mathrm{e}} \Delta(\Delta R / R)^{\text {Red-Ox }}$ \\
\hline 1 & 624 & 2.58 & 0.08 & 0.97 \\
2 & 1248 & 2.30 & 0.18 & -0.82 \\
3 & 1872 & 2.10 & 0.25 & -0.74 \\
4 & 2496 & 1.85 & 0.34 & -0.62 \\
5 & 3120 & 1.62 & 0.42 & -0.47 \\
6 & 3744 & 1.37 & 0.51 & -0.42 \\
7 & 4368 & 1.06 & 0.62 & -0.31 \\
8 & 4992 & 0.73 & 0.74 & -0.12 \\
\hline
\end{tabular}

${ }^{\mathbf{a}}$ Numbers 1 to 8 represent different deactivated POAP films. ${ }^{\mathbf{b}}$ Number of potential cycles to which each POAP film was subjected in the supporting electrolyte solution (scan rate: $0.01 \mathrm{~V} \cdot \mathrm{s}^{-1}$ ). ${ }^{\mathrm{c}}$ Voltammetric reduction charge of the different deactivated POAP films after being subjected to the number of potential cycles indicated in column 2 . d Degree of deactivation of each one of the POAP films after being subjected to the number of potential cycles indicated in column 2. The degree of deactivation was calculated from $\theta_{\mathrm{c}}^{\mathrm{d}}=1-\left(Q_{\text {Red,c }} / Q_{\text {Red,T }}\right)$, where $Q_{\text {Red,T }}\left(=2.8 \mathrm{mC} \cdot \mathrm{cm}^{-2}\right)$ is the voltammetric reduction charge of a nondeactivated film. ${ }^{\mathbf{e}}$ Relative surface resistance change at the reduced state of POAP at each degree of deactivation.

AR grade chemical were used throughout. o-Aminophenol (Fluka) was purified as described elsewhere [8, 9]. The solutions were prepared with water purified using a Millipore Milli-Q system. $\mathrm{NaClO}_{4}, \mathrm{HClO}_{4}, \mathrm{H}_{2} \mathrm{SO}_{4}$, $\mathrm{Na}_{2} \mathrm{SO}_{4}$, benzenesulphonic acid and sodium benzenesulphonate and $\mathrm{Cu}\left(\mathrm{ClO}_{4}\right)_{2}$ (Merck, ar grade) were used without further purification.

Ferric sulphate Mallinckrodt, analytical reagent grade, was employed in deactivation experiments.

\section{Results and Discussion}

\subsection{Electronic Transport in Thin Metal Films}

Electronic transport in thin metal films is strongly affected by interfacial phenomena. For example, the scattering of conduction electrons at planar interfaces defined by the top and bottom surfaces of the film under study can contribute significantly to the resistivity. In the case of thin metal films, the electrical resistivity $\rho_{\mathrm{f}}$ is higher than the bulk resistivity $\rho_{\mathrm{m}}$ of the massive metal of the same structure as the metal film and the $\rho_{\mathrm{f}} / \rho_{\mathrm{m}}$ ratio decreases with increasing the film thickness, $\phi_{\mathrm{m}}$. This "Size Effect" becomes evident when $\phi_{\mathrm{m}}$ is comparable with the mean free path, $l_{\mathrm{m}}$, of the conduction electrons. The theory to account for the size effect was postulated by Fuchs [20] and Sondheimer [21]. The exact expression for the dependence of the film resistivity, $\rho_{\mathrm{f}}$, as a function of $\phi_{\mathrm{m}}$ is complicated. However, it can be reduced to a limiting form when $\phi_{\mathrm{m}} / l_{\mathrm{m}} \geq 1$ : 


$$
\rho_{f} / \rho_{m}=1+(3 / 8)(1-r) l_{m} / \Phi_{m}
$$

In Equation (1), $r$ is the specularity parameter [21]. This parameter represents the probability of an electron being reflected specularly or diffusely at the film surface. The $r$ value ranges from 0 for complete diffuse scattering to 1 for complete specular scattering. At first, it should be considered that thin metal films can be prepared to satisfy the Fuch's model in a sufficient way to exhibit a specularity parameter near 1 (a surface with a smooth mirrorlike finish which is free of defects). However, this parameter, which is also interpreted as the fraction of the surface which specularly reflects electrons, depends on the quality of the metal film surface, that is, on the method of preparation of the metal film [2]. In this sense, an appreciable fraction of the conduction electrons can be scattered diffusely and give rise to an additional resistance, which correlates with roughness of surface topography and the presence of surface defects. All these imperfections should lead to experimental $r$ values lower than 1 .

Besides factors above mentioned if foreign entities are present on the film surface, translational symmetry parallel to the interface, changes and additional scattering of the conduction electrons occurs. This electron dispersion effect brought about by the presence of entities on the metal surface, thereby acting as dispersion centres for the surface reflection of the electrons from the inside of the metal, has been analysed on the basis of Equation (1). Assuming that the specularity, $r$, is the principal parameter influenced by the surface concentration of foreign scattering centres at the film surface $\Gamma_{\text {surf, }}$ differentiation of Equation (1) leads to the relationship:

$$
\Delta \rho_{f}=-3 / 8\left(\rho_{m} l_{m} / \Phi_{m}\right)(\Delta \mathrm{r})
$$

On the assumption that the increase of $\Gamma_{\text {Surf }}$ increases the diffuse scattering of the electrons, $\Delta r=-k \Gamma_{\text {Surf }}$, an increase $\Delta \rho_{\mathrm{f}}$ would be expected with increasing $\Gamma_{\text {surf }}$ (Equation (2)). In terms of the resistance changes $(\Delta R=$ $\Delta \rho_{\mathrm{f}} G / \phi_{\mathrm{m}}$ ), Equation (2) can be written as:

$$
\Delta R=-3 / 8 G\left(\rho_{m} l_{m} / \Phi_{m}^{2}\right) \Delta \mathrm{r}
$$

In the Wissmann's interpretation [22] the film resistivity change can be expressed in terms of the product of an apparent scattering cross section of the entity, $\sigma_{\mathrm{a}}$, and the density of scattering centres at the surface, $N_{\mathrm{a}}$ as:

$$
\Delta \rho_{f}=\left(\rho_{m} l_{m} / \Phi_{m}\right)\left(\sigma_{a} N_{a}\right)
$$

By comparing Equations (2) and (4), it is evident that a $\Delta r$ decrease can be associated to an increase in $\sigma_{\mathrm{a}}$.

\subsection{The gold Film Electrolyte Interface}

Figure 1 shows $\Delta R / R-E$ responses of a $30 \mathrm{~nm}$ thick gold film contacting solutions which contain $\mathrm{ClO}_{4}^{-}, \mathrm{SO}_{4}^{-2}$,

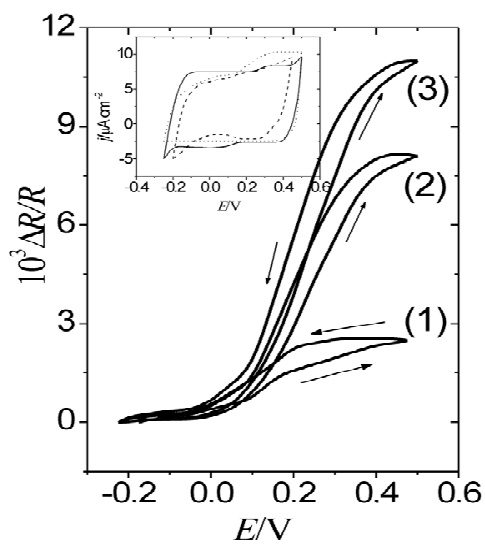

Figure. 1. $\Delta R / R$ vs. $E$ responses of a gold film electrode $\left(\phi_{\mathrm{m}}=\right.$ $30 \mathrm{~nm}$ ) contacting different electrolytes: (1) $0.1 \mathrm{M} \mathrm{HClO}_{4}+$ $0.4 \mathrm{M} \mathrm{NaClO}_{4}$; (2) $0.4 \mathrm{M} \mathrm{Na}_{2} \mathrm{SO}_{4}+0.1 \mathrm{M} \mathrm{H}_{2} \mathrm{SO}_{4}$; and (3) 0.4 $\mathrm{M}$ sodium benzenesulphonate $+0.1 \mathrm{M}$ benzenesulphonic acid. $v=0.01 \mathrm{~V} \cdot \mathrm{s}^{-1}$. Inset: the corresponding voltammograms.

and BS anions, respectively. In this case $\Delta R / R$ changes are referred to the potential value $E=-0.2 \mathrm{~V}$, where it is assumed the absence of specific adsorption of anions on gold.

The resistance change of gold films in the presence of weakly adsorbed anions, such as $\mathrm{ClO}_{4}^{-}$, has been ascribed to a surface charge effect rather than to a specific adsorption effect [4]. However, different resistance changes in the presence of anions able to act as surface impurities $\left(\mathrm{SO}_{4}^{-2}\right.$ and $\left.\mathrm{BS}\right)$ can be explained in terms of the apparent scattering cross sections of the adsorbates present on the metal film surface [22] (Equation (4)). In this connection, while the geometrical dimension of the BS anion lying flat on a plane is about $0.54 \mathrm{~nm}^{2}$ [23] that of sulphate is around $0.18 \mathrm{~nm}^{2}$ [24]. Besides, aromatic sulphonate anions exhibit distinguishable charged and non-charged parts (the sulphonate group and the benzene ring) as compared with the sulphate anion, which would lead to a stronger interaction of the high electron density of the benzene ring of the BS anion with metal surfaces. A strong adsorbability of (BS) anions on Au(111) singlecrystal electrodes, which inhibits the oxidation of the gold surface, was reported by Dutkiewicz and Skoluda [23]. Then, the sequence of $\Delta R / R$ changes $\left(\mathrm{ClO}_{4}^{-}<\right.$ $\mathrm{SO}_{4}^{-2}<\mathrm{BS}$ ) observed in Figure 1 for the three different anions can be considered as representative of a sequence of adsorbabily of these anions on the gold film electrode [4].

Figure 2 shows the resistance response corresponding to a $30 \mathrm{~nm}$ thick gold film electrode contacting a $\mathrm{Cu}(\mathrm{II})$ solution when the potential is decreased in the negative direction from $E=0.55 \mathrm{~V}$. Upd-ups processes of a copper monolayer on gold [19] occurs within the potential range 


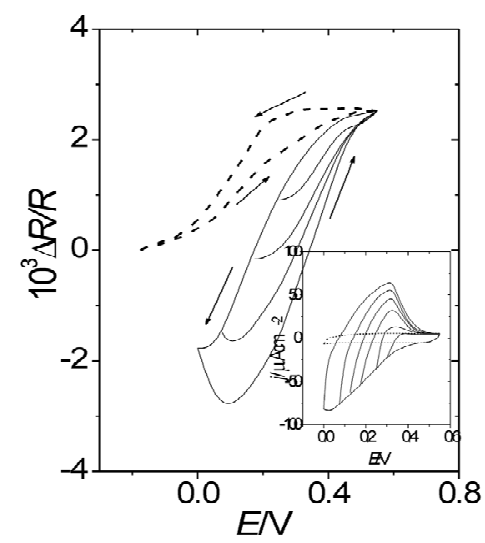

Figure. 2. (-) $\Delta R / R$ vs. $E$ response of a gold film electrode $\left(\phi_{\mathrm{m}}=30 \mathrm{~nm}\right)$ contacting a $0.1 \mathrm{M} \mathrm{HClO}_{4}+10^{-4} \mathrm{M} \mathrm{Cu}\left(\mathrm{ClO}_{4}\right)_{2}$ solution; (- - ) $\Delta R / R$ vs. $E$ response for the same gold film in the sole presence of the supporting electrolyte: $0.1 \mathrm{M} \mathrm{HClO}_{4}$. $v=0.01 \mathrm{~V} \cdot \mathrm{s}^{-1}$. Inset: the corresponding voltammetric responses. In the presence of $\mathrm{Cu}(\mathrm{II})$ the potential limit was gradually extended from $E=0.5 \mathrm{~V}$ up to $E=0.0 \mathrm{~V}$.

$0.0 \mathrm{~V}<E<0.55 \mathrm{~V} / \mathrm{SCE}$. At $E=0.55 \mathrm{~V}$ it is assumed that the copper momolayer is completely desorbed from the gold surface. Then, in this case an enough positive potential, such as $E=0.55 \mathrm{~V}$, was taken as a reference potential to assess the change of the resistance with increasing the copper coverage. As the potential is decreased towards the negative direction from $E=0.55 \mathrm{~V}$, the copper coverage increases and the gold film resistance decreases. For $E<0.0 \mathrm{~V}$, bulk copper deposition occurs [19]. Resistance decrease during copper upd process on gold was also observed by Hansen [25] and Rath [26]. This decrease suggests that the copper adlayer on the gold surface is more densely packed than the gold surface itself and then it could reflect electrons more specularly than the proper gold film surface.

\subsection{The Gold Film POAP Film Interface during the Redox Conversion of the Polymer}

Simultaneous $\Delta R / R-E$ and $j-E$ responses for the $30 \mathrm{~nm}$ thick gold film coated with POAP films of different thickness were recorded in the same electrolytes above mentioned $\left(\mathrm{ClO}_{4}^{-}, \mathrm{SO}_{4}^{-2}, \mathrm{BS}\right.$ and $\left.\mathrm{Cu}(\mathrm{II})\right) \cdot j-E$ responses at a given scan rate $(v)$ and POAP thickness in the presence of the different anions do not show big differences. This is shown in Figure 3 for the $Q_{\text {Red }}=1.5 \mathrm{mC} \cdot \mathrm{cm}^{-2}$ thick POAP film. In the inset of Figure 3, the corresponding response for a thinner POAP film $\left(Q_{\text {Red }}=0.22 \mathrm{mC} \cdot \mathrm{cm}^{-2}\right)$ is also shown.

$\Delta R / R-E$ responses for the gold film coated with the $0.12 \mathrm{mC} \cdot \mathrm{cm}^{-2}$ thick POAP film, in the presence of the different electrolytes, are compared in Figure 4. Figure 5 shows resistometric responses for other two thicker POAP films deposited on the same gold film (curves (a),

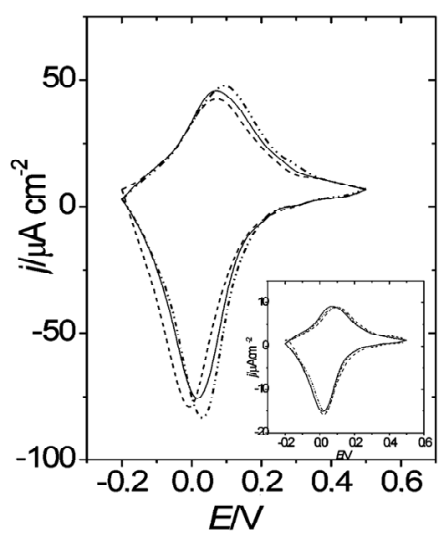

Figure 3. $j$ vs $E$ responses for a gold film $\left(\phi_{\mathrm{m}}=30 \mathrm{~nm}\right)$ coated with a $1.5 \mathrm{mC} \cdot \mathrm{cm}^{-2}$ thick POAP film. Electrolytes: (一) $0.1 \mathrm{M} \mathrm{HClO}_{4}+0.4 \mathrm{M} \mathrm{NaClO}_{4}$; (----) $0.4 \mathrm{M} \mathrm{Na}_{2} \mathrm{SO}_{4}+0.1$ $\mathrm{M} \mathrm{H}_{2} \mathrm{SO}_{4}$; and (-.. - .. -) $0.4 \mathrm{M}$ sodium benzenesulphonate + $0.1 \mathrm{M}$ benzenesulphonic acid. $v=0.01 \mathrm{~V} \cdot \mathrm{s}^{-1}$. Inset: $j$ vs $E$ responses for the same gold film coated with a $0.22 \mathrm{mC} \cdot \mathrm{cm}^{-2}$ POAP film.

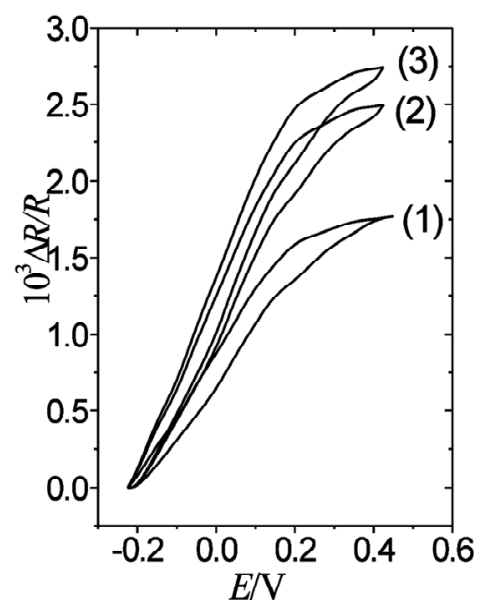

Figure. 4. $\Delta R / R$ vs. $E$ responses of a gold film electrode $\left(\phi_{\mathrm{m}}=\right.$ $30 \mathrm{~nm}$ ) coated with a $0.12 \mathrm{mC} \cdot \mathrm{cm}^{-2}$ thick POAP film in the presence of different electrolytes: (1) $0.1 \mathrm{M} \cdot \mathrm{HClO}_{4}+0.4 \mathrm{M}$ $\mathrm{NaClO}_{4}$; (2) $0.4 \mathrm{M} \mathrm{Na}_{2} \mathrm{SO}_{4}+0.1 \mathrm{M} \mathrm{H}_{2} \mathrm{SO}_{4}$; and (3) $0.4 \mathrm{M}$ sodium benzenesulphonate $+0.1 \mathrm{M}$ benzenesulphonic acid. $v=0.01 \mathrm{~V} \cdot \mathrm{s}^{-1}$.

$Q_{\text {Red }}=0.22 \mathrm{mC} \cdot \mathrm{cm}^{-2}$ and curves (b), $Q_{\text {Red }}=1.5$ $\mathrm{mC} \cdot \mathrm{cm}^{-2}$ ). In all cases $\Delta R / R$ changes were referred to the potential value $E=-0.2 \mathrm{~V}$, where POAP is in its reduced state.

Two facts can be remarked by comparing resistance responses of a gold film free of polymer (Figure 1) with $\Delta R / R$ changes of the same gold film coated with different POAP thickness (Figures 4 and 5). Firstly, the magnitude of the $\Delta R / R$ change, for a given anion, is strongly attenuated by the presence of the POAP film on the gold surface, as it is compared with the corresponding $\Delta R / R$ change of the gold film free of polymer. The attenuation is more pronounced as $\phi_{\mathrm{p}}$ increases. In this connection, 
considering the adsorption of BS anions on the gold film surface free of POAP (Figure 1), the $\Delta R / R$ change decreases nearly 90 per cent by the presence of a $1.5 \mathrm{mC} \cdot \mathrm{cm}^{-2}$ thick POAP film on the gold surface (Figure 5). Secondly, while distinguishable $\Delta R / R$ changes are even obtained for thin POAP films $\left(Q_{\text {Red }}=0.12\right.$ and 0.22 $\mathrm{mC} \cdot \mathrm{cm}^{-2}$ ) contacting the different anions, for the 1.5 $\mathrm{mC} \cdot \mathrm{cm}^{-2}$ thick POAP film the $\Delta R / R$ change becomes independent of the type anion present in the external solution. Both facts are represented in Figure 6, where $\Delta R / R-Q_{\text {Red }}$ dependence at $E=0.5 \mathrm{~V}$ (POAP in the oxidised state) is shown. As it can be seen from Figure 6, for $Q_{\text {Red }}>0.8$ $\mathrm{mC} \cdot \mathrm{cm}^{-2}$, the $\Delta R / R$ change becomes independent of both the POAP thickness and the anion contacting the polymer. However, it is also interesting to note that for $Q_{\text {Red }}<0.25$ $\mathrm{mC} \cdot \mathrm{cm}^{-2}$ (see also Figures 4 and 5) even when $\Delta R / R$

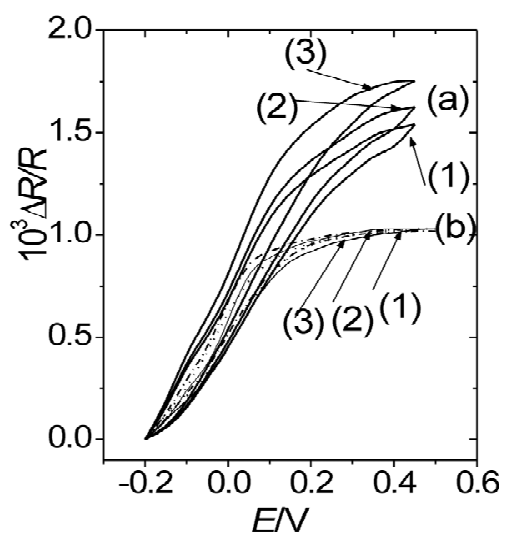

Figure. 5. $\Delta R / R$ vs. $E$ responses of a gold film electrode $\left(\phi_{\mathrm{m}}=\right.$ $30 \mathrm{~nm}$ ) coated with (a) a $0.22 \mathrm{mC} \cdot \mathrm{cm}^{-2}$ thick POAP film and (b) a $1.5 \mathrm{mC} \cdot \mathrm{cm}^{-2}$ thick POAP film, in the presence of same electrolytes indicated in Figure 4: (1) $0.1 \mathrm{M} \mathrm{HClO}_{4}+0.4 \mathrm{M}$ $\mathrm{NaClO}_{4}$; (2) $0.4 \mathrm{M} \mathrm{Na}_{2} \mathrm{SO}_{4}+0.1 \mathrm{M} \mathrm{H}_{2} \mathrm{SO}_{4}$; and (3) $0.4 \mathrm{M}$ sodium benzenesulphonate $+0.1 \mathrm{M}$ benzenesulphonic acid. $v=0.01 \mathrm{~V} \cdot \mathrm{s}^{-1}$.

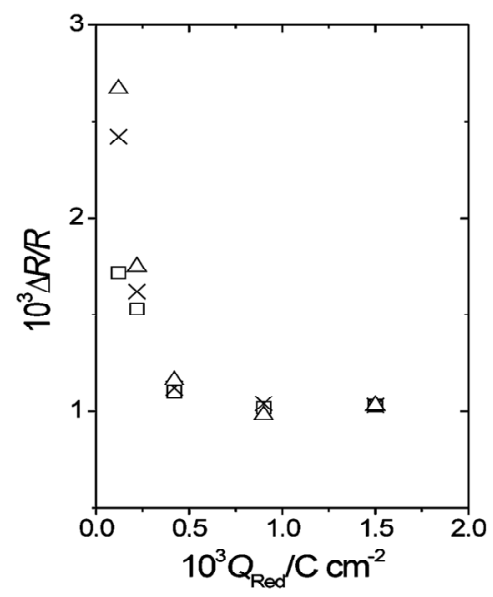

Figure. 6. $\Delta R / R$ vs. $Q_{\text {Red }}$ dependence at $E=0.5 \mathrm{~V}$ (POAP in the oxidised state). Symbols represent different anions: $(\Delta)$ BS; (X) sulphate; (O) perchlorate. changes for the different anions are attenuated as compared with the gold film surface free of polymer, they follow the same tendency for anion adsorption on gold, that is, $\mathrm{ClO}_{4}^{-}<\mathrm{SO}_{4}^{-2}<\mathrm{BS}$. Similar effects of the external electrolyte on the resistance response of gold films coated with very thin POAP films were observed in the presence of the $\mathrm{Cu}(\mathrm{II})$ cation. Figure 7 shows the $\Delta R / R-E$ response (curve (a)) corresponding to a $30 \mathrm{~nm}$ thick gold film contacting a $\mathrm{Cu}(\mathrm{II})$ solution within the potential range $0.0 \mathrm{~V}<E<0.55 \mathrm{~V}$. A strong attenuation of the $\Delta R / R-E$ response for the copper upd process on the gold film surface is observed when it is coated with POAP films (curves (b)-(d) in Figure 7). In this case the oxidised state of POAP $(E=0.55 \mathrm{~V})$ was taken as reference to assess the resistance decrease in going towards the negative potential direction.

As it can be seen, the $\Delta R-E$ response of the 1.5 $\mathrm{mC} \cdot \mathrm{cm}^{-2}$ thick POAP film contacting a $\mathrm{Cu}(\mathrm{II})$ solution (curve (d) in Figure 7) nearly coincides with the curve (e) within the potential range $0.0 \mathrm{~V}<E<0.5 \mathrm{~V}$. Curve (e) corresponds to a POAP film $\left(1.5 \mathrm{mC} \cdot \mathrm{cm}^{-2}\right)$ in the presence of perchlorate anion. Then, again it can be conclude that the $\Delta R / R-E$ response of a gold film coated with a thick POAP film is unique and independent of the external solution contacting the polymer film. This is also shown in the inset of Figure 7, where the $\Delta R / R-Q_{\text {Red }}$ dependence is represented at $E=0.0 \mathrm{~V}$, where POAP is in its reduced state. Again, for $Q_{\text {Red }}>0.8 \mathrm{mC} \cdot \mathrm{cm}^{-2}$ the resistance becomes a constant independent of the polymer thickness.

It is established, in accordance with the theory [27],

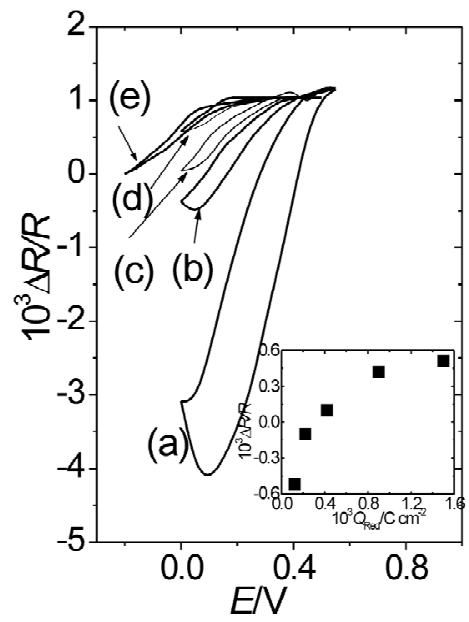

Figure 7. (a) $\Delta R / R$ vs. $E$ response of a gold film electrode $\left(\phi_{\mathrm{m}}\right.$ $=30 \mathrm{~nm}$ ) contacting a $0.1 \mathrm{M} \mathrm{HClO}_{4}+10^{-4} \mathrm{M} \mathrm{Cu}\left(\mathrm{ClO}_{4}\right)_{2}$ solution; $\Delta R / R$ vs. $E$ responses of the same gold film coated with different thickness of POAP: (b) $0.12 \mathrm{mC} \cdot \mathrm{cm}^{-2}$; (c) 0.22 $\mathrm{mC} \cdot \mathrm{cm}^{-2}$ and (d) $1.5 \mathrm{mC} \cdot \mathrm{cm}^{-2}$. Curve (e) corresponds to a POAP film $\left(1.5 \mathrm{mC} \cdot \mathrm{cm}^{-2}\right)$ in the presence of perchlorate anion. The same electrolyte above indicated. Inset: $\Delta R / R$ vs. $Q_{\text {Red }}$ dependence at $E=0.0 \mathrm{~V}$ (POAP in the reduced state). 
that the density of the polymer layer decreases with the film thickness, i.e., from the metal surface to the polymer solution interface. Internal parts of polymer films could have a different structure than their external parts. In this connection, during the synthesis of polymer films [28], two or more stages of the polymerisation process are usually distinguished: first, islands of the polymer are formed at the substrate surface, then a continuous film, which is compact (non-porous), is formed by fusion of these islands, and the further growth takes place above this compact layer giving an external porous part of the film. One should note that a continuous film is only formed after the end of the first stage of the polymerisation process. The technique of the surface resistance only detects processes occurring at the metal|polymer interface. Resistance measurements applied to gold film coated with POAP films seem to indicate that polymer coverage lower than $0.25 \mathrm{mC} \cdot \mathrm{cm}^{-2}$ are not sufficiently compact at the metal|polymer interface to prevent the specific adsorption of anions and cations proceeding from the external electrolyte on the gold film surface (Figures 47).

It is possible that at low POAP thickness $\left(<0.25 \mathrm{mC} \cdot \mathrm{cm}^{-2}\right)$ either polymer islands or polymer layers with imperfections exist on the gold surface. In this sense, high permeability values of some polymer films [29] to transport of species from the solution in contact with the polymer to the metal substrate have been attributed to dispersed polymer structures with imperfections (e.g., pinholes and large channels) with dimensions large compared to those of species present in solution. However, as the amount of polymer increases $\left(Q_{\text {red }}>0.8 \mathrm{mC} \cdot \mathrm{cm}^{-2}\right)$ the gold surface should be covered by a polymer film compact enough (fusion of islands or absence of imperfections) that the resistometric response (curve (b) in Figure 5 and curve (e) in Figure 7) can be considered due to processes at the metal polymer interface free of effects related to the interaction of species proceeding from the external electrolyte with the metal surface. In the last case (thick POAP films), the change of the gold film resistance in going from the reduced state of POAP to the oxidised one, could be explained only in terms of the generation of electronic entities at the polymer chains near the electrode surface, which occurs by electron transfer across the polymer metal interface. In this connection, the redox switching of POAP was interpreted in terms of the oxidation of the amino groups to imine [12]. Thus, it is not unreasonable to expect that imine sites act themselves as different scattering centres compared with amine sites, increasing in this way the diffuse reflection (increase of $\Delta R / R$ ) of conduction electrons on the gold surface during POAP oxidation. The increase of $\Delta R / R$ during POAP oxidation could also be explained in terms of an interfacial distribution of scatterers (imine sites) in the oxidised state with a spacing among them constant and larger than that corresponding to amine sites in the reduced state [1]. In this connection, during POAP oxidation only one in every four or five amine sites are converted to the corresponding imine sites [12], giving rise to gaps which eventually would yield a distribution of oxidised sites less compact than the corresponding distribution of reduced ones. Thus, a distribution of scatterers (oxidised sites) less compact than the distribution of reduced sites should lead to a more diffuse reflection (lower specularity parameter value in Equation (3)) of conduction electrons at the gold POAP interface [1], which in turn yields a resistance increase during POAP oxidation. Thus, the increase of the gold film resistance going from the reduced $(E=-0.2 \mathrm{~V})$ to the oxidized state $(E=0.5 \mathrm{~V})$ of POAP can be associated with a decrease of the specularity parameter of the gold film $r$ (Equation (3)), with increasing the degree of oxidation $\left(\theta_{\mathrm{OX}}\right)$ of the polymer film deposited on it $\left(\Delta r=-k \cdot \theta_{\mathrm{Ox}}\right)$. Then, Equation (3) can be written as

$$
\Delta R=-3 / 8 G\left(\rho_{m} l_{m} / \Phi_{m}^{2}\right) k \theta_{\mathrm{OX}}
$$

As can be seen from Equation (5), if $k$ is a constant independent of $\theta_{\mathrm{Ox}}$, an increase of $\Delta R$ with the increase of the degree of oxidation of the polymer film, should be expected. The degree of oxidation to the polymer can be defined at each potential, $E$, as by the ratio $\theta_{\mathrm{Ox}}=$ $Q_{\mathrm{Ox}} / Q_{\mathrm{T}, \mathrm{Ox}} \cdot Q_{\mathrm{Ox}}$ is the oxidation charge at eahc potential $E$, obtained from the voltammetric current recorded at a given scan rate and $Q_{\mathrm{T}, \mathrm{Ox}}$ is the voltammetric charge corresponding to the completely oxidized polymer film. Further confirmation about the different reflecting properties of the oxidised and reduced states of POAP can be found in the different values of the site interaction parameters $(p)$ obtained from the cathodic and anodic votammetric responses of POAP $[8,9]$. The following values of the anodic and cathodic site interaction parameters were reported for POAP, $p_{\mathrm{a}}=-0.55 \mathrm{M}^{-1}$ and $p_{\mathrm{c}}$ $=-0.18 \mathrm{M}^{-1}$, respectively. Both are negatives, thus involving a repulsive energy of interaction. As a higher repulsion is observed between oxidised sites than reduced ones, then a more extended configuration of oxidised sites should be expected than the corresponding distribution of reduced ones. Then, again, it would be expected that the distribution of oxidised sites reflects electrons more diffusely than the distribution of reduced ones.

Respect to the polymer redox conversion at the gold film surface, one has to keep in mind that the resistance changes at metal films are not the direct result of the electron transfer between the species on the metal film surface and the metal but they rather originate from the effect of foreign surface entities on the conduction electrons of the metal itself. At this point it should be pointed 
out that despite the creation of chemical bonds between the polymer and the metal film, the absolute value of the gold film resistance at a given thickness (for instance, $R$ $\sim 20 \mathrm{ohm}$ for $\phi_{\mathrm{m}}=30 \mathrm{~nm}$ ) does not change with POAP deposition, only the relative $\Delta R / R$ value, referred to either the reduced $(E=-0.2 \mathrm{~V})$ or oxidised $(E=0.5 \mathrm{~V})$ state of the polymer, varies with the potential scanning $(-0.2 \mathrm{~V}<E<0.5 \mathrm{~V})$ (see curve (b) in Figure 5). This should be indicative of a resistometric change only related to an interfacial (metal polymer) electron dispersion process occurring during oxidation-reduction of the polymer. On the contrary, generation of a nonconducting dead layer (subsurface impurity) during POAP deposition leading to a reduction of the metallic thickness should lead to an increase of the absolute value of the gold film resistance.

\subsection{Deactivation of POAP Films Studied by Surface Resistance}

As was indicated in the experimental section, POAP coated gold film electrodes were deactivated by soaking in a ferric cation solution. The reduction of the POAP electroactivity after contact with a ferric cation solution was attributed to the interaction of iron ions with the redox sites of POAP, which impedes the protonation reaction of the polymer [18]. Figure 8 shows simultaneous voltammetric $(j-E)$ and resistometric $(\Delta R / R-E)$ responses of a $30 \mathrm{~nm}$ gold film coated by a $2.8 \mathrm{mC} \cdot \mathrm{cm}^{-2}\left(\phi_{\mathrm{p}}=60\right.$ $\mathrm{nm})$ thick POAP film, in contact with the supporting electrolyte solution $\left(0.4 \mathrm{M} \mathrm{NaClO}_{4}+0.1 \mathrm{M} \mathrm{HClO}_{4}\right)$. These responses correspond to an immediately prepared POAP film. The POAP films maintain these response on potential cycling within the potential range $-0.2 \mathrm{~V}<E<$ $0.5 \mathrm{~V}$ up to 500 cycles. These POAP films are herein

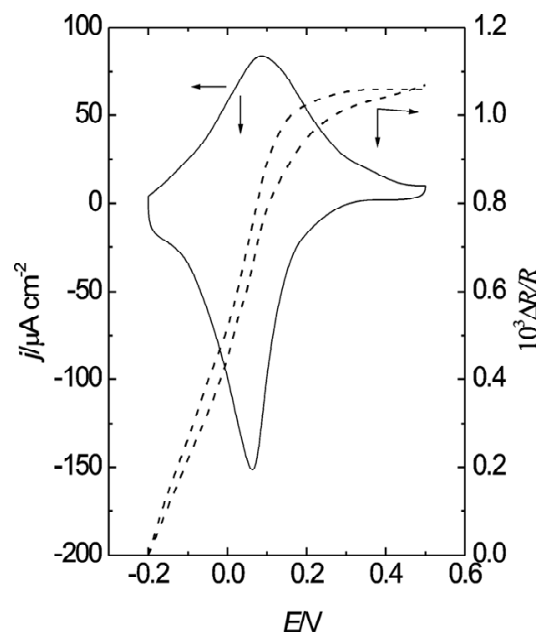

Figure 8. Simultaneous $\Delta R / R$ vs. $E$ and $j$ vs. $E$ responses of a $30 \mathrm{~nm}$ thick gold film coated with a $60 \mathrm{~nm}$ thick POAP film. Electrolyte: $0.4 \mathrm{M} \mathrm{NaClO}_{4}+0.1 \mathrm{M} \mathrm{HClO}_{4}$. Scan rate: $v=$ $0.01 \mathrm{~V} \cdot \mathrm{s}^{-1}$. called nondeactivated films. However, an attenuation of both voltammetric and surface resistance responses was observed when POAP films are soaked in a ferric cation solution. Deactivation experiments were performed with a series of 8 electrodes (see first column in Table 1), where each POAP film was soaked in a $0.1 \mathrm{M} \mathrm{H}_{2} \mathrm{SO}_{4}+$ $0.05 \mathrm{M} \mathrm{Fe}_{2}\left(\mathrm{SO}_{4}\right)_{3}$ solution for a different time period (see second column in Table 1). Then, each one of these electrodes was extracted from the solution containing ferric ions and it was copiously washed with the supporting electrolyte solution, then the electrode was again transferred to the electrochemical cell containing the supporting electrolyte solution. The corresponding $j-E$ and $\Delta R / R$ responses were again recorded for each one of the 8 POAP films. As was indicated, an attenuation of both $j-E$ (Figure 9) and $\Delta R / R$ (Figure 10) responses was observed after a POAP film contacts a ferric cation solution. These attenuations were considered indicative of the polymer deactivation.

Voltammetric reduction charge values corresponding to the completely reduced POAP films were compared for both nondeactivated films $\left(Q_{\text {Red,T }}=2.8 \mathrm{mC} \cdot \mathrm{cm}^{-2}\right)$ and films subjected to the soaking process in the presence of the Fe(III) solution ( $\left.Q_{\text {Red,c }}\right)$ (see column 3 in Table 1); then, a degree of deactivation (column 4 of Table 1) was defined as

$$
\theta_{\mathrm{c}}=1-\left(Q_{\mathrm{Rad}, \mathrm{c}} / Q_{\mathrm{Rad}, \mathrm{T}}\right)
$$

where $Q_{\text {Red,c }}$ is the total reduction charge assessed by integration of the corresponding $j-E$ response from $E=$ $0.5 \mathrm{~V}$ towards the negative potential direction for a deactivated film and $Q_{\mathrm{Red}, \mathrm{T}}=2.8 \mathrm{mC} \cdot \mathrm{cm}^{-2}$ is the total reduc-

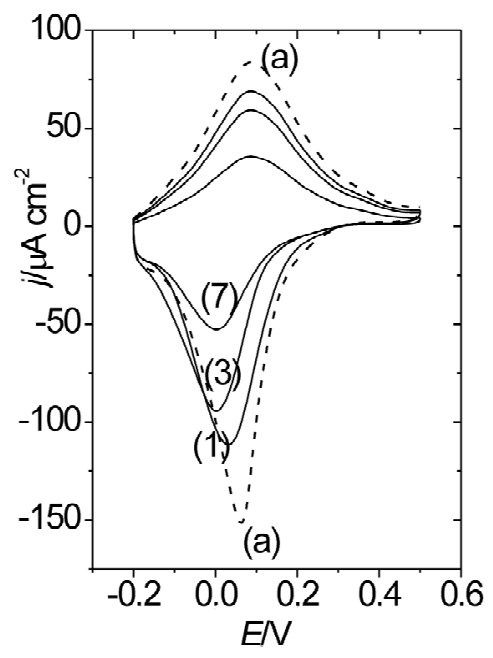

Figure 9. Voltammetric $(j-E)$ responses for a $2.8 \mathrm{mC} \cdot \mathrm{cm}^{-2}$ $\left(\phi_{\mathrm{p}}=60 \mathrm{~nm}\right)$ thick POAP film. (a) A nondeactivated POAP film $\left(\theta_{c}=0\right)$. Curves (1), (3) and (7) correspond to films whose degree of deactivation is: $\theta_{\mathrm{c}}=0.13 ; \theta_{\mathrm{c}}=\mathbf{0 . 3 3}$ and $\theta_{\mathrm{c}}=$ 0.60, respectively (Table 1). Electrolyte: $0.1 \mathrm{M} \mathrm{HClO}_{4}+0.4$ $\mathrm{M} \mathrm{NaClO}_{4}$. Scan rate: $v=0.01 \mathrm{~V} \cdot \mathrm{s}^{-1}$. 


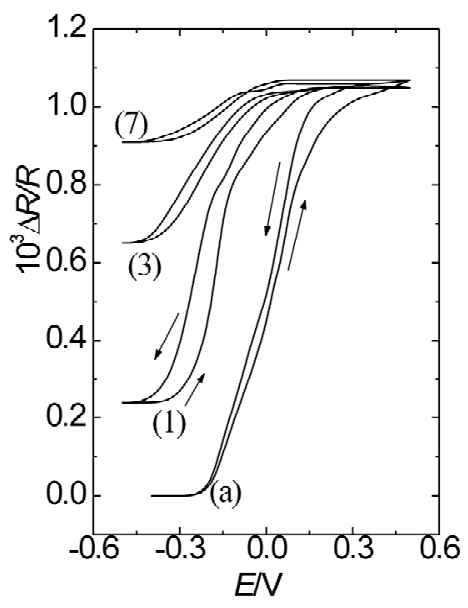

Figure 10. $\Delta R / R-E$ responses obtained simultaneously with the $(j-E)$ responses shown in Figure 9. The same notation and conditions as in Figure 9 have been used.

tion charge for the non-deactivated film. In this way, taking $Q_{\text {Red,T }}=2.8 \mathrm{mC} \cdot \mathrm{cm}^{-2}$ as reference charge. However, values of $\theta_{c}>0$ are indicative of POAP films that have been deactivated.

With regard to surface resistance, one can see that starting from $E=0.5 \mathrm{~V}$ towards the negative potential scan direction, an attenuated decrease of the resistance response is observed as compared with that of an immediately prepared film. Also, the higher $\theta_{\mathrm{c}}$ is, the more attenuated the resistance response becomes. The attenuated resistance decrease at the reduced state of $\operatorname{POAP}(E=$ $-0.3 \mathrm{~V}$ ), starting from $E=0.5 \mathrm{~V}$, can be attributed to a decreasing amount of active amine redox sites as the polymer becomes deactivated more, which in turn leads to the creation of inactive gaps within the redox site configuration of POAP at the metal/polymer interface. The $\Delta R / R$ value, at the constant potential value of $E=-0.30$ $\mathrm{V}$, was represented as a function of $\theta_{\mathrm{c}}$ for the eight films listed in Table 1 (Figure 11). In terms of distribution of scatterers at the polymer metal interface, the increase in $\Delta R / R$ at $E=-0.3 \mathrm{~V}$ with increasing $\theta_{\mathrm{c}}$ should indicate the existence of redox sites distributions which reflect conduction electrons more diffusely than redox configuretions of an immediately prepared film. This would indicate that the distance between adjacent redox sites becomes larger as the polymer film becomes deactivated more. However, if only the number of active redox sites of POAP were decreased (keeping their distribution constant) with increasing deactivation, only one slope should be obtained. As can be seen from Figure 11, a $(\Delta R / R) / \theta_{\mathrm{c}}$ slope about of $1.82 \times 10^{-3}$ is obtained within the range $\theta_{\mathrm{c}}$ comprised between 0.0 and 0.45 . However, the $(\Delta R / R) / \theta_{\mathrm{c}}$ $\left(=0.80 \times 10^{-3}\right)$ slope decreases for $\theta_{\mathrm{c}}$ values higher than 0.45 . The decrease of the slope at high degrees of deactivation $\left(0.45<\theta_{c}<0.74\right)$ should be indicative of the existence of a redox sites distribution relatively more

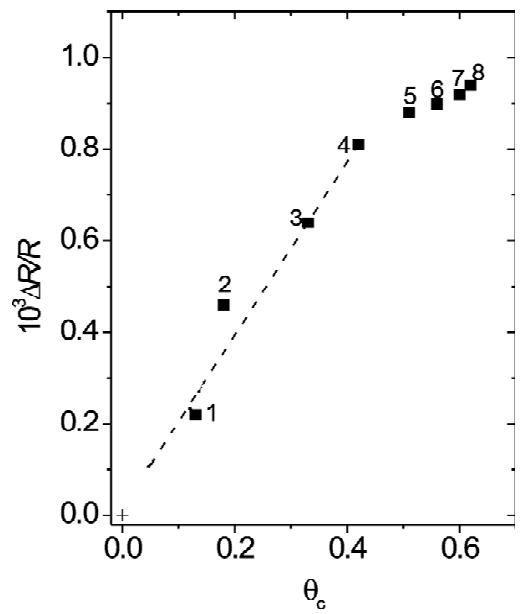

Figure 11. $\Delta R / R$ vs. $\theta_{\mathrm{c}}$ dependence for deactivated films. Each number corresponds to each one of the 8 POAP films listed in Table $1 . \Delta R / R$ values extracted at the potential value $-0.30 \mathrm{~V}$. Electrolyte: $0.1 \mathrm{M} \mathrm{HClO}_{4}+0.4 \mathrm{M} \mathrm{NaClO}_{4}$. Scan rate: $v=0.01 \mathrm{~V} \cdot \mathrm{s}^{-1}$.

compact at high $\theta_{\mathrm{c}}$ values, as compared with the distribution at $\theta_{\mathrm{c}}$ values lower than 0.45 .

As was indicated in the experimental section, a second deactivation method of POAP films was employed in this work. That is, POAP films were also deactivated by prolonged potential cycling in a supporting electrolyte solution. The loss of electroactivity of a polymer film under continuous potential cycling has been attributed to an excessive uptake of doping sites by dopant anions from the external electrolyte solution. Then, after a great number of potential cycles, anions may associate strongly with positive sites of the polymer chains resulting in electrostatic cross-linking, which in turn leads to the polymer deactivation [30]. In this regard, another series of eight POAP-coated gold film electrodes was prepared (see first column in Table 2) and each one of them was subjected to a different number of potential cycles (higher than 500 cycles, see second column in Table 2) within the potential region $-0.2<E<0.5 \mathrm{~V}$ in a deoxygenated supporting electrolyte solution. Then, the corresponding $j-E$ responses for each one of the eight POAP films were recorded. Again, an attenuation of both voltammetric (Figure 12) and surface resistance (Figure 13) responses was observed for these films when the number of potential cycles was higher than 500 .

Again, a degree of deactivation, $\theta_{\mathrm{c}}$, was defined on the basis of Equation (5). The total reduction charge $Q_{\text {Red,c }}$ assessed by integration of the corresponding $j-E$ response from $\mathrm{E}=0.5 \mathrm{~V}$ towards the negative potential direction (Figure 12) for a deactivated film and the corresponding $\theta_{\mathrm{c}}$ values are listed in columns 3 and 4 , of Table 2, respectively.

Figures 12 and 13 show voltammetric and resistometric responses for a nondeactivated film and films (5); (7) 


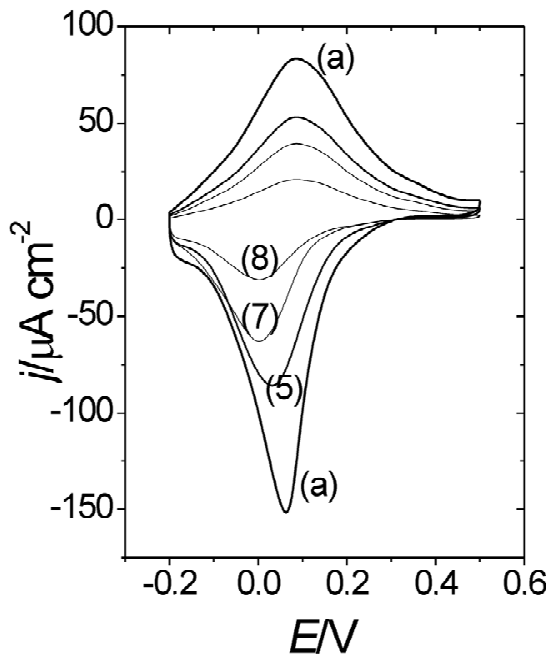

Figure 12. Voltammetric $(j-E)$ responses for a $2.8 \mathrm{mC} \cdot \mathrm{cm}^{-2}$ $\left(\phi_{\mathrm{p}}=60 \mathrm{~nm}\right)$ thick POAP film. (a) A nondeactivated POAP film $\left(\theta_{c}=0\right)$. Curves (5), (7) and (8) correspond to films whose degree of deactivation is: $\theta_{\mathrm{c}}=0.42 ; \theta_{\mathrm{c}}=0.62$ and $\theta_{\mathrm{c}}=$ 0.74, respectively. Electrolyte: $0.1 \mathrm{M} \mathrm{HClO}_{4}+0.4 \mathrm{M}$ $\mathrm{NaClO}_{4}$. Scan rate: $v=0.01 \mathrm{~V} \cdot \mathrm{s}^{-1}$.

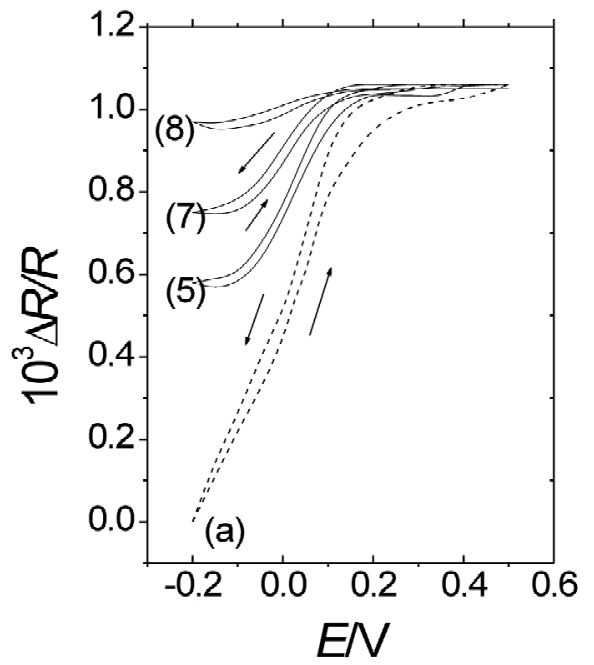

Figure 13. $\Delta R / R-E$ responses obtained simultaneously with the $(j-E)$ responses shown in Figure 12. The same notation and conditions as in Figure 12 have been used.

and (8), respectively, indicated in Table 2. Figure 14 shows a nearly linear $(\Delta \mathrm{R} / \mathrm{R})$ versus $\theta c$ dependence with a slope, around $1.25 \times 10^{-3}$ within the whole $\theta \mathrm{c}$ range. This linear dependence shown in Figure 14 is different from that shown in Figure 11, for POAP films deactivated by contact with a ferric cations solution, where two different slopes are observed. The existence of only one slope for POAP films deactivated by prolonged potential cycling is indicative of a distribution of redox sites which increases with a constant spacing between active sites all along the deactivation process $\left(0<\theta_{\mathrm{c}}<0.74\right)$. As the slope value $\left(1.25 \times 10^{-3}\right)$ for deactivated films by pro-

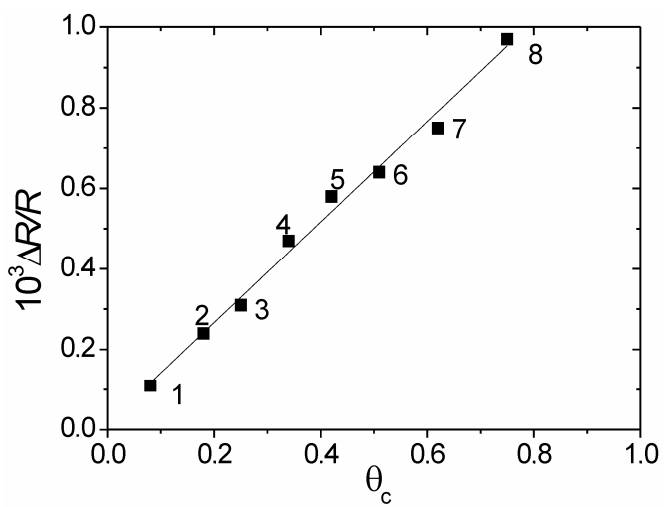

Figure 14. $\Delta R / R$ vs. $\theta_{\mathrm{c}}$ dependence for deactivated films. Each number corresponds to each one of the 8 POAP films listed in Table 2. $\Delta R / R$ values extracted at the reduced state of POAP. Electrolyte: $0.1 \mathrm{M} \mathrm{HClO}_{4}+0.4 \mathrm{M} \mathrm{NaClO}_{4}$. Scan rate: $v=0.01 \mathrm{~V} \cdot \mathrm{s}^{-1}$.

longed potential cycling is intermediate between those values obtained for deactivated films by contact with a ferric cation solution, it is possible to consider the former distribution of redox sites exhibits an intermediate distance between adjacent sites as compared those observed for POAP deactivated by soaking in a ferric cation solution.

\section{Conclusion}

Poly(o-aminophenol) films of different thickness between 0.12 and $1.5 \mathrm{mC} \cdot \mathrm{cm}^{-2}$ were supported on a thin gold film whose thickness was of the order of the mean free path of conduction electrons of gold. A dependence of the gold film resistance on the external electrolyte composition was observed during the redox conversion of poly(o-aminophenol) films whose thicknesses was lower than $0.25 \mathrm{mC} \cdot \mathrm{cm}^{-2}$. It seems to be indicative of a competition, at the gold film surface, between the redox process of the polymer and adsorption of different species contained in the electrolyte. This would reflect a discontinuous character of polymer thickness lower than $0.25 \mathrm{mC} \cdot \mathrm{cm}^{-2}$ on the gold surface. The gold film resistance becomes independent of both external electrolyte composition and polymer film thickness for polymer thickness higher than $0.8 \mathrm{mC} \cdot \mathrm{cm}^{-2}$. Thus, the gold film resistance only depends on the degree of oxidation of the polymer film for thicknesses higher than $0.8 \mathrm{mC} \cdot \mathrm{cm}^{-2}$. The resistance change for thick POAP films deposited on gold was assigned to the own redox transformation of the polymer, free of adsorption effects of species proceeding from the electrolyte solution. Thus, polymer thickness higher than $0.8 \mathrm{mC} \cdot \mathrm{cm}^{-2}$ seems to be compact enough at the metal polymer interface to prevent the interaction of the species contained in the supporting electrolyte with the gold film surface. Surface resistance measurements were also employed to study the POAP deactivation. 
POAP films were deactivated employing two different methods: immersion in a ferric cation solution and prolonged potential cycling. The interpretation of the surface resistance data on the basis of the electron scattering caused by species present on a metal film surface, allowed one to detect the existence of different distributions of scatterers at the gold film/POAP film interface according to the deactivation method employed. While only one distribution of redox sites was observed for POAP deactivated by prolonged potential cycling within the whole degree of deactivation range $\left(0<\theta_{c}<0.74\right)$, two distributions with different spacing among redox sites were detected for POAP deactivated by immersion in a ferric cation solution.

\section{Acknowledgements}

The author gratefully acknowledges to the Consejo Nacional de Investigaciones Científicas y Técnicas (CONICET) and also to the Facultad de Ciencias Exactas, National University of La Plata (UNLP).

\section{REFERENCES}

[1] J. W. Geus, "Chemisorption and Reaction on Metallic Films,” In: J. R. Anderson and G. Hohler, Eds., Vol. 1, Academic Press, London, 1971, p. 388,

[2] D. Schumacher, "Surface Scattering Experiments with Conduction Electrons," In: J. R. Anderson and G. Hohler, Eds., Vol. 128, Springer Tracts in Modern Physics, Springer, Berlin, 1992, p. 67

[3] R. Tucceri, "A Review about the Surface Resistance Technique in Electrochemistry," Surface Science Reports, Vol. 56, No. 3-4, 2004, pp. 85-157. doi:10.1016/j.surfrep.2004.09.001

[4] R. I. Tucceri and D. Posadas, "Surface Conductance Study of the Anion Adsorption on Gold," Journal of Electroanalytical Chemistry and Interfacial Electrochemistry, Vol. 191, No. 2, 1985, pp. 387-399. doi:10.1016/S0022-0728(85)80031-0

[5] F. M. Romeo, R. I. Tucceri and D. Posadas, "Surface Conductivity Changes during the Electrochemical Adsorption of UPD Layers on Silver and Gold," Surface Science, Vol. 203, No. 1-2, 1988, pp. 186-200. doi:10.1016/0039-6028(88)90203-8

[6] R. I. Tucceri and D. Posadas, "Resistive Behavior of Thin Gold Film Electrodes under Direct Current Polarization," Journal of the Electrochemical Society, Vol. 130, No. 1, 1983, pp. 104-107. doi:10.1149/1.2119631

[7] R. I. Tucceri and D. Posadas, "Theoretical Approach to the Resistive Behavior of Thin Solid Film Electrode under Direct Current Polarization," Journal of the Electrochemical SocietyVol. 128, No. 7, 1981, pp. 1478-1483. doi:10.1149/1.2127667

[8] C. Barbero, J. J. Silber and L. Sereno, "Formation of a Novel Electroactive Film by Electropolynerization or Orthoaminophenol, Study of Its Electrochemical Structure and Formation Mechanism. Electropolymerization of Analogous Compounds," Journal of Electroanalytical Chemistry and Interfacial Electrochemistry, Vol. 263, No. 2, 1989, pp. 333-352. doi:10.1016/0022-0728(89)85103-4

[9] C. Barbero, J. J. Silber and L. Sereno, "Electrochemical Properties of Poly(o-Aminophenol) Modified Electrodes in Aqueous Acid Solutions," Journal of Electroanalytical Chemistry and Interfacial Electrochemistry, Vol. 291, No. 1-2, 1990, pp. 81-101. doi:10.1016/0022-0728(90)87179-N

[10] C. Barbero, J. Zerbino, L. Sereno and D. Posadas, “Optical Properties of Electropolymerized Orthoaminophenol," Electrochimica Acta, Vol. 32, No. 4, 1987, pp. 693697. doi:10.1016/0013-4686(87)87063-9

[11] S. Kunimura, T. Ohsaka and N. Oyama, " Preparation of Thin Polymeric Films on Electrode Surfaces by Electropolymerization of Orthoaminophenol," Macromolecules, Vol. 21, No. 4, 1988, pp. 894-900. doi:10.1021/ma00182a007

[12] R. I. Tucceri, C. Barbero, J. J. Silber, L. Sereno and D. Posadas, "Spectroelectrochemical Study of Poly-o-Aminophenol," Electrochimica Acta, Vol. 42, No. 6, 1997, pp. 919-927. doi:10.1016/S0013-4686(96)00277-0

[13] C. Barbero, R. I. Tucceri, D. Posadas, J. J. Silber and L. Sereno, "Impedance Characteristics of Poly-o-Aminophenol Electrodes," Electrochimica Acta, Vol. 40, No. 8, 1995, pp. 1037-1040. doi:10.1016/0013-4686(94)00373-9

[14] F. J. Rodríguez Nieto and R. I. Tucceri, "The pH Effect on the Charge Transport at Redox Polymer-Modified Electrodes. An AC Impedance Study Applied to Poly(oAminophenol) film Electrodes," Journal of Electroanalytical Chemistry, Vol. 416, No. 1-2, 1996, pp. 1-24. doi:10.1016/S0022-0728(96)04704-3

[15] A. Bonfranceschi, A. Pérez Córdoba, S. Keunchkarian, S. Zapata and R. Tucceri, "Transport Acorss Poly(o-Aminophenol) Modified Electrodes in Contact with Media Containing Redox Active Couples. A study Using Rotating Disc Electrode Voltammetry," Journal of Electroanalytical Chemistry, Vol. 477, No. 1, 1999, pp. 1-13. doi:10.1016/S0022-0728(99)00368-X

[16] R. I. Tucceri, "Redox Mediation and Permeation Process at Deactivated Poly (o-Aminophenol) Films. A Study Applying Rotating Disc Electrode Voltammetry and Electrochemical Impedance Spectroscopy," Journal of Electroanalytical Chemistry, Vol. 633, No. 1, 2009, pp. 198206. doi:10.1016/j.jelechem.2009.05.014

[17] R. Tucceri, "Charge Transfer and Charge Transport Parameters at Deactivated Poly(o-Aminophenol) Film Electrodes. A Study Applying Electrochemical Impedance Spectroscopy," Journal of Electroanalytical Chemistry, Vol. 659, No. 1, 2011, pp. 83-91. doi:10.1016/j.jelechem.2011.05.005

[18] J. Yano, H. Kawakami, S. Yamasaki and Y. Kanno, "Caption Capturing Ability and the Potential Response of a Poly(o-Aminophenol) Film Electrode to Dissolved Ferric Ions," Journal of the Electrochemical Society, Vol. 148, No. 2, 2001, pp. E61-E65. doi:10.1149/1.1339235

[19] A. Aramata, "Underpotential deposition, ," In: J. O. M. Bockris, R. E. White and B. E. Conway, Eds., Modern 
Aspects of Electrochemistry, Vol. 31, Plenum Press, New York, 1997, Chapter 4, p. 112.

[20] K. Fuchs, "Electrical Resistance in Thin Metal Films," Proceedings of the Cambridge Philosophical Society. Mathematical and Physical Sciences, Vol. 34, No. 1, 1938, pp. 100-193.

[21] E. H. Sondheimer, "Mean Free Path of Electrons in Metals," Advances in Physics, Vol. 1, No. 1, 1952, pp. 1-42. doi:10.1080/00018735200101151

[22] P. Wissmann, "The Electrical Resistivity of Pure and Gas Covered Metal Films," Zeitschrift für Physikalische Chemie, Vol. 71, No. 2, 1970, pp. 294-315.

[23] E. Dutkiewicz and P. Skoluda, "Adsorption of Benzenesulphonate Anions at an $\mathrm{Au}(111)$ Electrode: Application to Study of the Reconstruction Phenomena of the Au(100) Surface," Journal of the Chemical Society, Faraday Transactions, Vol. 92, No. 20, 1996, pp. 3763-3767. doi: $10.1039 / \mathrm{ft} 9969203763$

[24] "Tables of Interatomic Distances and Configurations in Molecules and Ions. The Chemical Society," Burlington House, London, 1958.

[25] W. N. Hansen, "Electrode Resistance and the Emersed Double Layer," Surface Science, Vol. 101, No. 1-3, 1980, pp. 109-122. doi:10.1016/0039-6028(80)90602-0
[26] D. L. Rath, "Studies of the Electrode Resistance in the Electrochemical Cell," Journal of Electroanalytical Chemistry and Interfacial Electrochemistry, Vol. 150, No. $1-2,1983$, pp. 521-534. doi:10.1016/S0022-0728(83)80232-0

[27] P. G. De Gennes, "Polymers at an Interface; a Simplified View," Advances in Colloids and Interface Science, Vol. 27, No. 1, 1987, pp. 189-209.

[28] G. Inzelt, M. Pineri, J. W. Schultze and M. A. Vorotyntsev, "Electron and Proton Conducting Polymers: Recent Developments and Prospects," Electrochimica Acta, Vol. 45, No. 15-16, 2000, pp. 2403-2421. doi:10.1016/S0013-4686(00)00329-7

[29] T. Ikeda, R. Schmehl, P. Denisevich, K. Willman and R. W. Murray, "Permeation of Electroactive Solutes through ultrathin Polymeric Films on Electrode Surfaces," Journal of the American Chemical Society, Vol. 104, No. 10, 1982, 2683-2691. doi:10.1021/ja00374a001

[30] P. Novák, K. Muller, K. S. V. Santhanami and O. Haas, "Electrochemically Active Polymers for Rechargeable Batteries," Chemical Reviews, Vol. 97, No. 1, 1997, pp. 207-281. doi:10.1021/cr9411810 